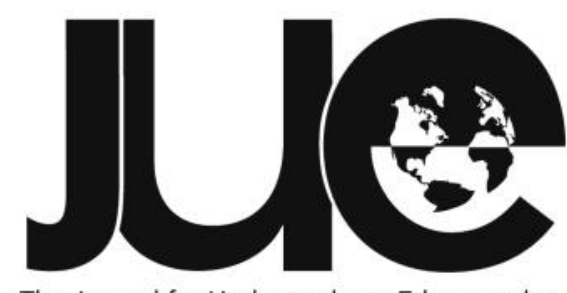

The Journal for Undergraduate Ethnography

\title{
“Don't Be a Son of a Bitch, Stand Up and Get What You Need": Understanding Italian Young Adults' Identity through Insults
}

\section{Eleonora Colzani}

Saint Louis University-Madrid Campus, eleonora.colzani@slu.edu

\section{ABSTRACT}

This qualitative study, grounded in the ethnography of communication, aims to describe how Italian young adults construct their individual and group identity through the use of insults. The existing literature has extensively investigated the communicative practice of insults, but no research has been done so far on insults in relation to the construction of identity in Italy. Therefore, this study enriches the literature, taking a linguistic, communicative, and pragmatic approach to the topic. In order to collect data, I conducted participant observation, one focus group, and three individual interviews. I applied thematic analysis to analyze the data. By interpreting members' meanings, I identified four main functions of insults: construction of identity through gender; construction of identity depending on social context; construction of identity through the light use of insults; and construction of identity through the offensive use of insults. 
nsults are a fundamental part of many world languages. As a matter of fact, Charles Flynn (1976) argued for the "universality" of certain kinds of insults, underlying the historical and geographical extent to which they are commonly used (3). As with every other component of speech, insults are subject to the influence of culture and context. Because of this influence, they are used in many different instances, in a huge variety of ways, and with countless different meanings. The habit that people have developed to hear and perhaps use insults on a regular basis should not lead to the assumption that insults are not worth examining. On the contrary, the ubiquity of insults across so many cultures and communities should provoke a spark of curiosity towards understanding the processes underlying their application, and their relative meanings in a specific cultural context.

The existing literature provides a comprehensive account of the use and nature of insults, describing and analyzing a variety of cultural settings and approaches, among them the ethnography of communication, content analysis, textual analysis and linguistic studies. Even though the existing studies provide undoubtedly a substantial contribution, no study has been conducted so far on the native perspective on the use of insults in Italy, and, more specifically, on the impact of insults on Italians' identity. In order to enrich the existing literature, the aim of this study is to analyze from the natives' point of view how the perception and evaluation of insults construct both individual and group identity among Italian young adults. Moreover, this study represents a guide for non-natives of the Italian speech community to understand the underlying meaning of insults and their relation to personal and communal identities. The research question that the study aims to answer is: How do Italian young adults construct and perform their own individual identity and their group identity through the interpretation and evaluation of insults?

In order to be able to answer it from the participants' points of view, this study is grounded in the ethnography of communication (EoC). This theoretical framework calls for the understanding of communicative phenomena exclusively from the natives' points of view. The data was gathered through interviews, participant observations and one focus group. The process of data analysis relied on the method of thematic analysis. More specifically, I constructed the analysis around Hymes' SPEAKING model, guided by symbolic interactionism as a sensitizing theory. From the data collected and analyzed through thematic analysis, four themes that describe how identity is constructed through the use of insults emerged: constructed depending on gender identity, constructed depending on social context, constructed through the light use of insults, and constructed through the offensive use of insults. The article concludes by summarizing and contextualizing these findings within the existing literature.

\section{Literature Review: Insults and Identity}

Numerous studies have already been conducted on the use of insults in different cultural settings, such as smaller, local speech communities (Murray 1979; Alderete et al. 2012), and larger, more general speech communities (Flynn 1976; Samarin 1969; Tartamella 2016). The existing literature provides an initial framework for studying the nature of insults in the field of communication studies, and has presented both quantitative and qualitative studies on the matter. Tartamella (2016)'s quantitative study analyzes the frequency of use of insults and the popularity of specific ones. Some qualitative studies focus on the use of insults through the lenses of linguistics, while others apply an ethnographic lens, looking at the association of insults to behavior and concepts. Generally speaking, the underlying theme of identity through the existing literature is apparent, if not explicitly specified. 
Vito Tartamella, an Italian scholar and journalist, has conducted various studies on the use of swearwords in Italian. In his book Parolacce (2016), he identifies 301 main swearwords that are commonly used in the country. However, in an online article, he points out that it is impossible to give a specific number that precisely clarifies the amount of swearwords that exist in the Italian dictionary, "both because new swearwords are continuously appearing (from slang, for instance), and because every word could become a swearword, if one gives it a derogatory meaning" (Tartamella 2011, n.p., my translation; unless otherwise noted, all translations from Italian are my own). In his long-term and extensive research, he identifies five main categories in which he divides the 301 most-used swearwords by type: sexual (39.2\%), religious (19.7\%), emphatic (14.9\%), insults (14.4\%), and excrement-related (11.6\%) (Tartamella 2016). The contribution of these quantitative studies, however, is limited, as it does not speak to the use and relative consequences of these terms. On the contrary, other studies, which were not conducted in the Italian context, opted for a qualitative approach, investigating the members' meanings in various defined speech communities.

Numerous studies have tried to define the nature of insults used in relation to a certain behavior or concept. An example is provided by Alderete et al.'s (2012) research on racial insults and the practice of smoking in Jujuy, Argentina. The most common relation analyzed in the literature is, however, that between insults and sexuality (Flynn 1976; Murray 1979; Samarin 1969). Previous research has approached this connection from various angles, giving the impression that the topic has been adequately explored. A common variable among all these studies is the focus on, or awareness of, the existence of cultural differences, and the impact of these differences on the use, interpretation, and actual meanings of insults. Flynn (1976) focuses on the types, the universality, and the diverse grounds for sex-related insults; Murray (1979) carries out a comparative study between the Black speech community and the gay community on the art of insulting, with an emphasis on ritual insults; and Samarin (1969) presents a comprehensive ethnographic study on the use of insults among the Gbeya, a tribe from Central African Republic. The completion of these works led to the relevant conclusion that there is a close relation between insults and "a person's physical characteristics" (Samarin 1969, 324), more specifically "direct or indirect references to genitals" (Flynn 1976, 3). Moreover, Flynn's study, which is more general in nature, lists several sex-related situations which are either considered insulting in themselves, or are motives to provoke insults, such as adultery and incest. Some of these reasons overlap with Murray's study (1979) focused on gay communities, among whom sex seems to be pivotal "in the corpus of gay ritual insults" (216). Another pattern that emerges from the existing literature is research on the way insults are used, always in relation to specific contexts. Among the various identified characteristics, expression of feelings, judgement, and retort were the most relevant ones. Nonetheless, the literature points out that insults have experienced a historical change in both meaning and usage (Flynn 1976; Kegl 1994; Murray 1979).

The literature connects the concept of insults to that of identity both implicitly, focusing more on the behavioral premises and consequences linked to the use of insults, and explicitly, by investigating how the use of insults constructs and performs identity meanings typical of a defined speech community. In Rosemary Kegl's textual analysis (1994), for instance, insults, or "abominable terms" are used as lenses to understand the political structure and underlying identity of Windsor's middle class (274). From a more ethnographic perspective, Alderete et al. (2012) study the effect of racial insults and ethnic discrimination on the integrity of identity among indigenous youths in Argentina, and its relation to their tendency to smoke. For these researchers, the integrity of identity refers to different factors that build up one's true and unique self, and that are threatened by external discrimination. The relation between identity and insults could equally well be understood in connection to sexuality. As for gay communities, sexual orientation is a huge part of their individual and group identity. Insulting a person's sexual orientation or anything related to it would be 
considered a direct threat to their identity and the group's identity. However, Murray (1979) suggests that "younger, liberationist-influenced gay men generally refrain from making insults about sexual activity and sexual identity," concentrating instead on "'tackiness', 'piss elegance' and closetry" (218). As for the sexrelated grounds for insults identified by Flynn (1976), illegitimacy - by which he means a rupture in the social construction of legitimacy is clearly connected to the concept of identity. As claimed in the study, "the questioning of someone's legitimacy is akin to questioning the basic sources of identity" (8-9). The link between identity and insults is therefore unquestionable.

Context and identity construct and, in turn, are constructed by the use of insults, where insults are understood as a communicative practice. According to Witteborn and Huang (2017), "analyzing and comparing communicative practices, which are always culturally situated, is essential for understanding social and political life in a particular locale" (142). Therefore, context and identity are, in turn, connected to the concept of culture, which, according to Philipsen (1992), "refers to a socially constructed and historically transmitted pattern of symbols, meaning, premises, and rules" about communicative conduct (7). Culture is a code of communication. It follows, then, that insults, as communicative practice and conduct, are characterized by a specific set of rules, norms, and premises which are highly dependent on the specific context in which they are used, and are worth analyzing to understand the social construction of the particular speech community that uses them.

\section{Conceptual Framework}

This study is grounded in the theoretical framework of the ethnography of communication, which aims to understand each particular communicative phenomenon exclusively from the natives' points of view. Initially developed by Dell Hymes in the 1960s, the ethnography of communication was formerly called the ethnography of speaking. Despite the different titles, "both expressions seem to refer to the same approach and body of work" in numerous studies (Philipsen and Couto 2005, 356). This study understands the
EoC to include both verbal and non-verbal communication. The EoC approach views "speaking as a culturally distinctive activity," and, therefore, worth analyzing (Tracy 2013, 53). It focuses on "the use of language in all its modes," meaning verbal and non-verbal forms of communication, situated "in the social life and the cultural system of particular speech communities" (Philipsen and Couto 2005, 355). The specific culture of the studied speech community must be, if not directly understood, at least acknowledged by the researcher, as "the terms for talk that reside within any one speech community are often culturespecific" (Wilkins 2009, 64). Acknowledging the importance of culture, EoC applies the SPEAKING model, devoted to the analysis of discourse understood as a series of speech acts in cultural context (Hymes 1972). It is an acronym that stands for:

- Scene and Setting, referring to time and space;

- Participants, referring to whoever is involved in the speech act;

- Ends, meaning the ultimate purpose of the act;

- Act Sequence, including form and content;

- Key, also known as the tone and seriousness;

- Instrumentalities, which refers to the style;

- Norms, related to the rules that affect the speech act; and

- Genre, commonly understood as the type of speech.

EoC underlines the importance of the cultural and situational context of a given communicative event when attempting to analyze it. In fact, as Witteborn and Huang (2017) effectively summarize, “EoC attempts to understand locally situated and culturally meaningful communicative practices through which people organize social life" (144).

Drawing from EoC, this study aims to analyze the meanings attributed by Italian young adults to the use of insults (means of speaking) in specific cultural, communicative events. In order to achieve an understanding of the 
members' meanings, which are "interpretative constructions assembled and conveyed by the ethnographer" (Emerson, Fretz, and Shaw 2011, 129), In this study, I employed symbolic interactionism as a sensitizing theoretical framework around which I organized both research and analysis (Tracy 2013). As part of its foundation, symbolic interactionism complements EoC, functioning therefore as a useful sensitizing theory in the context of this study. Symbolic interactionism, developed by George H. Mead and Hebert Blumer, "focuses on the symbolic dimensions of human communication," trying to understand "how meaning and identity are co-created through interaction" (Tracy 2013, 51).

\section{Methods}

This study aims to describe, through participants' meanings, how the use of insults as terms of address among Italian young adults constructs their group and individual identities. In this research, the process of data analysis relies on the method of thematic analysis, which aims for the identification of themes and patterns in the data. This method represents the best choice in this study, not only because it offers "a more accessible form of analysis," but also because it "works both to reflect reality and to unravel the surface of "reality"' (Braun and Clarke 2006, 81). In order to understand the meanings that natives attribute to insults in order to construct their identities, the researcher must be able to go beyond the surface of the discourse produced by participants. Thematic analysis allows for this necessary process. In the following sections, all the applied methods to collect data are presented in detail; then, the data analysis is extensively described.

\section{Participant Observation}

Participant observation allows the researcher to gain important data through the observation of and, in certain cases, direct interaction with participants. As Tracy (2013) states, “participant observation is valuable precisely because it reveals the multi-faceted nature of the scene" (76). The few interactions that I was able to observe resulted in primary sources of examples of in vivo terms and native uses, which I would not have been able to observe otherwise. Mainly, these interactions were conversations among Italians picked up on the street or observed in bars in Madrid. I did not always know the participants in these conversations, limiting the background data available on them. An important exception is represented by a participant observation session conducted with a group of four male Italian young adults, aging 21 to 24, during a night out in Madrid. This observation lasted for several hours, providing sufficient demographic and background information. Moreover, since I was an insider to the culture, I was able to provide my own insights and guide my observations in an effective way. As Tracy (2013) cautions us, an insider to a culture runs the risk of going native and losing track of the research purpose. During the data collection process, I did my best to keep the study's research questions in mind and to focus on trying to answer them from my participants' points of view. Another limitation that may have affected the study is the impossibility of filming the interviews conducted in order to record the exact words, voice tone, and body language expressed by the participants. Rather, for most of the interviews I recorded only the audio, and managed to write down notes only at a later time. Tracy (2013) argues that "memories can still serve as useful data" (116). Therefore, I made a conscious effort to be as accurate and thorough as possible when recording my thoughts and memories of the interactions observed.

\section{Individual interviews}

Individual interviews are an incredibly fruitful method of gaining meaningful data from a study's participants. Defined as "guided question-answer conversations," interviews allow for a mutual exchange of ideas, due to their intimate and flexible nature (Tracy 2013, 131). In fact, as Tracy (2013) exemplifies, "interviewer and interviewee are, in many ways, conversational partners" (132). The friendly atmosphere granted by interview sessions allows the researcher to acquire data that would be otherwise hard to access with different methods.

The choice of an appropriate sampling strategy is pivotal for the ultimate efficacy of this method, as it "is integral to answering the research questions" (Tracy 2013, 152). For this study, I conducted 3 individual interviews, 
which lasted 20 to 30 minutes each. In order to effectively choose the appropriate participants for this study, I combined two sampling plans described by Tracy (2013): convenience sampling and typical instance sampling. I interviewed 3 Italian young adults: Stefano, male, age 24, college student; Luca, male, age 24, full-time personal trainer; and Giulia, female, age 23, college student. I conducted the interviews in Italian. I opted for unstructured interviews, which resulted in the acquisition of unexpected data because the interviewees were mostly in control of the conversation. All the individual interviews were conducted through Skype calls, due to the geographic limitation. Being a talkative couple, the participants were able to carry on naturally occurring discussions among themselves with little need for me to moderate them. Unfortunately, mediated interviews suffer a number of disadvantages, among them the presentation of "mediocre embodied and nonverbal data" (Tracy 2013, 165). However, despite these potential limitations, the data that I elicited from my participants via Skype was rich and useful to answer my research question, after I triangulated it with other data sets. I also conducted a face-to-face interview with two participants, a married young couple: Serena, female, age 30, beautician; and Marco, male, age 32, metalworker. The dynamic of the focus group, despite the limitation imposed by the limited number of participants, was more than satisfying.

\section{Data Analysis}

This study relies on thematic analysis to analyze the collected data. Thematic analysis is a widely used method "for identifying, analyzing, and reporting themes within data" (Braun and Clarke 2006, 79). After collecting data, I proceeded through the method's six phases of analysis, as described by Braun and Clarke (2006): (i) familiarizing with the data, (ii) generating initial codes, (iii) searching for themes, (iv) reviewing themes, ( $v$ ) defining and naming themes, and (vi) producing the report (87). Respecting the "recursive process" of thematic analysis (Braun and Clarke 2006, 86), the primary codes generated during the second phase have been revised multiple times, as well as the themes identified in the following phase. The primary coding process resulted in 33 initial codes. Once repeated for the sake of clarity, the same coding process resulted in 55 different codes. The first two phases of the analysis were conducted in Italian, which was easier and less confusing. Once the coding process was brought to an end, the whole set of data was translated into English. In the end, five potential themes were identified (see table 1 in the Appendix). Each of these potential themes included multiple initial codes. For instance, the theme depends on included codes such as: technical college (istituto tecnico), high school (liceo), boss, subordinates, and friends. The huge disparity among the codes made it necessary to create sub-themes, which would help organize the codes into more meaningful groups (see Table 1 and Table 2 in the Appendix).

At the end of the analysis process, four main themes were identified (see Table 2 in the Appendix), concluding phase (v): 1. construction of identity through gender; 2 . construction of identity depending on social context; 3. construction of identity through the light use of insults; and 4. construction of identity through the offensive use of insults. These themes, constructed through the interpretation of members' meanings, provide an answer to the study's research question. In the following sections, all the patterns are described through the use of exemplars.

\section{Construction of Identity through Gender}

Throughout the interviews, various participants underlined the connection between the use of insults and gender identity. While Flynn's study (1976) focused on insults in relation to "sexual non-conformity," "adultery," "illegitimacy," and "homosexuality" (4-9), the data collected in this study uncover another sphere of the world of insults and sexuality/gender. As recent work has argued, gender identities are not a given, but are constructed through different types of communication. As Kiesling (2001) points out, "gender should [..] be thought of as [..] a fluid, cultural construction by social actors who use language to 'do gender"' (250). A clear distinction emerges from the data between how the use of insults constructs individual identity and group identity. The use of insults at 
the personal level performs, and is proper of, masculine identity. As Marco, male, explained in the focus group (and I have translated all participants' quotes from Italian to English): “I think that the use of insults is only a men thing. I mean, it's not that only the men say 'bitch' or 'asshole' or whatever. But I would expect more a man saying words like that than a woman."

Reasoning on "participants" - in the sense that Hymes (1972) uses the term in his model the interviewee explicitly links the use of insults to the concept of gender. He establishes a division between men and women when it comes to the use of insults: insults construct an identity typical of males, namely masculinity, and while women can use insults, Marco explains that they are not expected to. Individuals characterized by a masculine identity, therefore, are the only acceptable "participants" in communication acts that involve insults. From this premise, it follows that the use of insults is a means of performing masculine identity, and accordingly, they construct it. On the contrary, feminine identity is performed through the non-use of insults. Insults can, therefore, be considered as a linguistic strategy implemented by people to create and define different identities. As Stefano, male, elaborated in his interview: "A woman must be delicate like a flower. A woman is a flower. Delicate and impeccable. Insults are the opposite of this. Who the hell would hear something like, 'Yo bitch, what's up', and be like 'She is so delicate'. Come on."

The interviewee implies that insults are not an appropriate means of speaking to construct feminine identity. On the contrary, the intrinsic characteristics of insults are oppositional to those of the fairer sex. Looking at Marco's and Stefano's comments, it seems to be the case that the use of insults constructs a masculine identity on the basis of perceived strength and confidence. Another interviewee, Serena, female, reinforces this point of view stating that "insults are typical of men. I rarely use them in conversation. It doesn't feel right." Serena reinforces Stefano's point of view, which implies that feminine identities are constructed through the non-use of insults, giving a sense of aesthetic perfection, fragility, and elegance that insults would take away. As someone with a feminine identity, Serena rejects the use of insults, even though she acknowledges that she also uses them, although rarely. Therefore, two distinct identities constructed by the use of insults, or lack thereof, are recognized: masculine identity, where strength and confidence are communicated through the use of insults, and feminine identity, where fragility and elegance are expressed through the nonuse of insults. Despite of this premise, however, some participants gave examples of women also using insults. Giulia's words during an interview illustrate this claim: "To me, insults are not connected to the gender of the person who's talking. If a guy says 'son of a bitch' it's the same as if a girl says it. Nothing changes to me. Maybe in the past people would have said that the girl was too sboccata (foul-mouthed), but today that's not the case anymore."

Giulia's comment illustrates a different norm that regulates the use of insults: a norm that appears to contradict the use of insults as a means to construct masculinity. Interestingly enough, Giulia said that "in the past" people would have supported Stefano's and Marco's perspective. The term sboccata clearly supports the past division between masculine and feminine identity, as it is used only when referring to women that inappropriately use insults. In the past, "gender performances [were] thus understood within a particular interpretive frame [..] about what kinds of interaction are typical of members with culturally relevant identities" (Kiesling 2001, $251)$. In this case, in the past it was believed that men had to carry out a masculine identity, and women had to carry out a feminine identity only. Gender identities were, therefore, already given.

Not only does the use of insults construct one's individual identity, but also one's individual identity contributes to determining the overall identity of the group of people in which one is interacting. These data analyses illustrate that within a specific speech community there may be multiple ways of speaking that compete with each other. As Hymes (1974) explains, a speech community is an "organization of diversity" (433), and accordingly, these contradictory norms for the use of insults are part and parcel of social life. 
On one hand, a community may value gender identities in a traditional way, understanding them in a frame where men have to carry out a masculine identity and women a feminine identity only. On the other hand, a community may be more progressive, believing that men and women are no longer characterized respectively by masculine and feminine identities. The following example applies to the former case.

If a man that genuinely believes in respecting traditional gender identities is having a conversation with a woman that rejects this social and communicative construct, he would feel uncomfortable carrying on a conversation in which insults are used. In order to exemplify this meaning, I constructed a "vignette" using data that I collected and pieced together (Tracy $2013,208)$. In the vignette, $M$ is the man that follows a traditional social construction of gender identities, and $F$ is the woman who does not. $\mathrm{M}$ and $\mathrm{F}$ are friends, and they just met by chance in the mall. After going to a party together the previous Saturday, M asks what happened after he left, as he heard bad news about something David did that night.

M: Hi, F! How is it going? What happened Saturday by the way?

F: Hey, M! Don't even ask. That asshole, David, ruined the party. What a fucking idiot.

M: Is it necessary to use that kind of language? But yeah, I heard. I'm sorry about that!

\section{F: He deserves what he deserves.}

In this communicative "scene," the man points out that the woman should not use this kind of language, clearly referring to insults. This study interprets that this is due to the conflicting way gender identities are perceived. Through the conservative masculine identity to which he subscribes, M perceives F's use of insults as a violation of the social norm that regulates interactions between masculine and feminine identities. As woman, $F$ should not have used insults, as it is not allowed in the already-given feminine identity. When looking for the social norm that guides a particular speech community, one must keep in mind that "while gender is in theory infinitely fluid, in the practice of most people's lives, it is constrained by cultural models" (Kiesling 2001, 267).

\section{Construction of Identity depending on Social Context}

The data present a clear pattern for insults to be dependent on different social contexts when it comes to their use. Not only individual gender identity is constructed through the use of insults, but also other factors, in relation to insults, appears to be pivotal in the process of shaping individual identities according to participants' perceptions: age, context, level of education, and workplace environment.

Age

Despite the relatively young age of this study's participants, a pattern emerged relating to the differences in using and interpreting insults as a younger teenager and as a young adult. For the sake of clarity, I estimate that the age that differentiates young adults and teenagers is 20 , while the teenage period goes from 15 to 20 years old. Participants associated these different age groups with different feelings and meanings motivating the use of insults. The young adults who were interviewed for this research hinted at the fact that a teenager's use of insults leads to, and aims at, two identityrelated outcomes: feeling older and feeling more secure. As Giulia, 23, reported in her interview:

The giovani (young people) between 15 and 20 years old use insults way too much, both in a light way and in an offensive way. When you are younger, the use of an insult could make you feel older and securer than just being polite and lovely. I'm not sure why, but that's the sensation that it [the use of insults] gives you.

Both the identity-related outcomes are clearly mentioned in Giulia's comment. These are not, however, final, tangible outcomes. They affect identity as sensations, abstract influences that seem to be volatile and highly situational. Youths' usage of insults to enhance their selfconfidence implies that they have a strong desire for self-confirmation and imposition. In a way, this practice could aim to reduce the difference in maturity and reliability that characterizes the two age groups through teenagers' speech practices. The purpose, then, of the use of insults among younger teenagers is to feel older, more mature, and, therefore, more confident. Moreover, according to Giuia's 
interpretation of the youth's speech community, the use of insults allows its "participants" to create a performative identity based on feeling more adult. Giulia does not clarify, however, what kind of identity the use of insults would construct among adults. Given the differentiation she makes between the two speech communities, it could be interpreted that adults' creative use of insults would say something different about their status and identities.

\section{Context}

From my participants' stories and opinions, it emerged that the outcomes of the use of insults is highly situational, and both their proper and improper use in certain situations constructs group identity. As Samarin (1969) wrote, quoting Hymes (1964): "it is indeed 'not the case that anyone can say anything, by any means, in any manner, to anyone else, or any occasion, and to any purpose"' (323). In particular, two meaningful social contexts emerged from the data: kind of academic education and workplace. From this analysis, it emerges that participants believe in the existence of a correlation between given identities, such as technical school student or manual worker, and the use of insults.

\section{Academic Education}

Participants underlined how through the subconscious analysis and interpretation of other people's insult-using practices, one shapes his or her own perceptions of the other people's social status. As Luca pointed out:

I think education has a saying in this [use of insults]. I mean, not what you study, but where. For example, when you go to a high school, you don't hear as many insults. But if you go to a technical school, they use so many insults and swearwords, without even a reason. Without thinking.

The identities and social statuses of hypothetical interlocutors are shaped in the participant's mind by their speech practices, namely the recurrence of insults in their speech. Their continuous use locates the interlocutor in a lower and less educated social group ("technical school students"), while seldom and responsible use grants the interlocutor a place in a higher and more educated social group ("high school students"). "Scene and setting" seem to play a fundamental role in the participant's reasoning, influencing the "key" as well. Regardless of the distinction between these two kinds of schools, which does not pertain to this study, it is remarkable that Luca believes that two speech communities exist, and they have different norms when it comes to the use of insults. This point is supported by Serena, who went to a vocational school. She thinks that "It [use of insults] is also related to education." Again, the use of insults in interactions influences the perceived identities of all interlocutors, especially the perceived social status.

\section{Insults in the Workplace}

In the same way social statuses are actively shaped by insult-use in interactions, the perceived social importance of interlocutors is also actively constructed by the use of insults. An important premise about the "scene and setting" is given by Marco, a metalworker, which mentioned that "the use of insults depends also on the job that you have." This is echoed by Giulia, who used to work in primary schools. She said that "In the education field, no one uses insults [...] Every context has its way of being." This is already indicative of group identity. Once again, different speech communities have different speech codes, and in order to be part of those, one has to share and use them; otherwise, the group's identity would be threatened. Another pattern that emerged in this context is that of how insults shape identities in the workplace hierarchy. As Marco said: "I call my colleague 'asshole, come here!' I mean, we are used to this. It's not offensive, it's more like a nickname, but not really. However, I don't do this with everyone. I wouldn't do this for sure with my boss. But with your subordinates it's fine."

"Scene," "setting," and "key" clearly influence the way insults construct identities in this context. The hierarchy is clearly stated by the participant: boss, me, and my subordinates. The use of insults is regulated by strict "norms" in this context, and breaking them would be a direct threat to the worker's identity and the group identity. The boss can use insults, but not receive them. The subordinates cannot insult their superiors, but they can insult each other. 
Therefore, one can assume that the use of insults not only shapes individual identities, but also it influences the dynamics of group identities: the unpunished use of insults is possible only among peers and subordinates, while the same communicative behavior with people in a higher position bears potentially negative consequences.

\section{Construction of Identity through Light Use of Insults}

A division between light and offensive insult use emerged almost instantaneously from the data collected. The different use of insults relates, according to my participants' interpretations, to two different ways of constructing identity. In order to proceed with the interpretative description of the data, it is fundamental to report the participants' distinction between light insults and offensive insults. Specifically, as Marco pointed out, "there are different levels of insults, and some are more delicate then others." Marco is referring to what Hymes (1972) named "key" in his model. According to the collected data, insults such as scema (foolish), stupida (stupid), and cretino (idiotic) are considered light insults, while figlio di puttana (son of a bitch) and troia (whore) are viewed as offensive insults. As already encountered previously, the "key" together with "scene and setting" appear to be fundamental in the understanding of the use of insults, its outcomes, and its "norms." An example that Serena provided is that she "would never say tonta (very dumb) to a friend of mine. I rarely use insults, but I would maybe say scema." The use of an insult belonging to the inappropriate level in a certain context would, in fact, threaten both the individual and group identity, as it disrupts the commonly intelligible "norm."

The light use of insults appears to be acceptable in interpersonal communication, to the extent that Marco defines it as "a habit", and Luca claims that "in a friendly manner it is more than accepted." There are, however, some rules that regulate this specific use. Marco explains this by saying: "You don't use insults with everyone. You do it with friends, or with people that at least you know more or less well. Especially if you do it jokingly, you need to have confidenza (to be close to someone) with that person." This interpretation sheds light on the "key" (jokingly), the "participants" (friends), and the premises (confidenza) shaping the "norms" in this communicative "genre." Therefore, the use of light insults, if carried out properly, constructs a friendly group identity. In other words, friendship is performed through the light use of insults. It follows that in this realm insults do not have an intrinsically bad meaning. Among friends, the use of insults represents a validation and a consolidation of the already existing connection between the people involved. As Luca confirms, "if an insult is used jokingly by a loved one, I take it with a smile." The reaction to being addressed with an insult, therefore, is not understood as hateful communication, with which insults are widely associated, but as a term proper to the speech community that performs the overall identity. An interesting exception is pointed out by Luca, who says that "son of a bitch remains a serious insult." The other participants, however, did not mention whether this or other offensive insults are accepted within the context of light use of insults.

However, a striking contradiction within the data is to be noted. The fact that some contradictions figure among the data is not surprising or alarming. In fact, As Philipsen and Coutu (2005) point out, "many types of means and meanings can be found in a given community" (369). Therefore, contradictions are, if not a given, absolutely normal. During our interview, Marco declared at different times both that using insults does not constitute a habitual practice for him, as was mentioned, but also that their light use is "a habit." Some possible explanations for this disparity in his comments are the context of use, namely "scene and setting", the people involved in the conversation, namely "participants", i.e. friends or non-friends, and the intended meaning behind the insult, namely "end" and "instrumentalities", e.g. joke, negative judgement, defensive reaction, or friendly response.

Overall, in the context of the light use of insults, the data derived from participant observation seem to support the claim that the appropriate light use of insults is a habitual practice, and it performs a friendly group 
identity. The data that follow were recorded during a night-out in a bar ("scene and setting") between five friends ("participants"). When, during that evening, a man named Giacomo realized that he forgot his tobacco at the hotel, and therefore that he would be forced to ask around for cigarettes the whole night as he does not like his friends' tobacco brands, he insulted himself. Another friend, Stefano, soon noticed, however, that a man standing close to their group of friends had the same brand of tobacco Giacomo smokes. Not being able to speak English well, Giacomo asked his friends to go and ask him for some tobacco. Elia and Stefano reacted to this request with two different forms of insults:

G: Shit, I left my kit at home. I'm such an idiot.

$\mathrm{E}$ : Well, take some of mine then.

G: No fucking way! That shit sucks.

S: That man smokes your same kind of tobacco. Go and ask for some.

G: (Tempted but insecure expression).

E: What now?

G: I am ashamed of my English, you know that! Why don't you ask?

E: Don't be the son of a bitch (figlio di puttana) like you always do. If you want it, you stand up and go ask for it. (Jokingly irritated).

S: You are such a jerk (coglione) when you act like this! (Laughing).

G: Whatever.

Elia used the term son of a bitch, while Stefano addressed Giacomo as a jerk. Despite these terms seeming to appear more offensive than other kinds of insults, they are undeniably implemented in a friendly environment without negative consequences to the harmony of the group. The habit of use is underlined by the fact that when Giacomo first insulted himself for the mistake he had made, no one reacted by trying to console him. The situation was, in fact, perceived as a normal one, and not a tragic or exceptional one. Moreover, the degree of friendship shared among the five friends is showed by how the insults are applied as buffers on Giacomo's situation. The speech code of the group was not threatened by the use of these insults, as the use complied with the group's norms. Insults were, therefore, used in an appropriate way, constructing and performing a friendly group identity.

\section{Construction of Identity through the Offensive Use of Insults}

Most notably, insults are not only used in a light way, but also in an offensive way. The offensive use of insult is perceived to be radically different from a light one by the study's participants. As previously mentioned, the light use of insults helps construct relationships such as friendship, enhancing the group's identity. The offensive use of insults, on the contrary, leads to the construction and performance of an oppositional identity, in contrast with the other speaker during an interaction. Luca, for instance, gave this specific example:

This happened last week, more or less, during a soccer match. There was a face-off between me and an opponent, and he pushed me down. Then, he looked at me and said 'Stand up pussy'. I snapped back at him right away something like 'Shitty asshole, shut up that you cannot handle this anymore.'

The use of insults in this example is undoubtedly offensive. The aim, however, is not limited to offence. The use of "pussy" and "shitty asshole" seem to aim to distance one person from the other. There is nothing that associates the two men interacting. They both want to make this clear by underlying the weaknesses or negative characteristics of the others that they do not share. This way of using insults constructs, therefore, an oppositional identity. It can also be considered as a defense mechanism. This interpretation is also supported by Serena, who commented that "I use insults when I am talking about someone that did something wrong to me, like 'what a bitch'." In her words, the underlying meaning is related to creating a distance between her and the other person by insulting her. The group identity constructed through this use of insults, therefore, is a negative, oppositional, and defensive one. 


\section{Conclusion}

Generally speaking, identity and communication are directly related (Littlejohn, Foss, and Oetzel 2016). Through the analysis of the data collected by giving "close attention to the terms or phrases that members regularly use" (Emerson, Fretz, and Shaw 2011, 142), I found that this statement also holds true when identity is linked to insults. Identity is, in fact, constructed and performed through the use of insults in various ways, all of which are highly dependent on context. The members' meanings investigated during this study suggest four different ways in which identity is constructed through the use of insults. First of all, identity is built depending on gender. The interpretation of the use of insults constructs and leads to the performance of both masculine and feminine identities, which are extremely oppositional to each other. When insults are taken to be an acceptable communicative practice, a more masculine identity is shaped. In fact, those embodying masculine identities can use insults; they are almost expected to do so. On the contrary, feminine identities are shaped by the non-use of insults. This perceived identity at the individual level also constructs group identities. For instance, when a man with a masculine identity interacts with a woman that rejects feminine identity, their perspectives would be at clash. On the contrary, when two women who reject feminine identity communicate, they would not clash, enhancing the group identity. For outsiders, it is fundamental to understand the existence and the difference between these two identities in order to recognize it and respect it.

Identity is constructed depending on the social context. Three sub-themes were identified: age, education and social status, and workplace and social importance. The use of insults varies according to the age group to which a person belongs: youths of 15-20 years old or young adults aged 20-35. The data collected allowed the construction of the meaning of the young adults' perspectives of how youths use insults. According to my participants' perspectives, youths use insults to shape their individual identity towards feeling older and more secure. Participants also hinted that, through the subconscious analysis and interpretation of other people's insult-using practices, one shapes his or her own perceptions of the other people's social statuses. This was applied to the field of education, meaning that a more frequent and irresponsible use of insult shapes a lower social status. Last but not least, a relation between identity, insults, and perceived social importance emerged during the study. The use of insults, in fact, both constructs and is constructed by the hierarchy of a specific workplace. The relation between speech code norms and solid group identity is undeniable. This study tries to unveil these norms in order to provide a description of how these affect the strength and dynamic of the group's identity.

In addition, identity through the use of insults is constructed specifically through the light use of insults and offensive use of insults, which lead to oppositional outcomes. The light use of insults is a habitual and potentially harmless practice if conducted properly. It underlines closeness between individuals. Understanding the non-offensive feature of insults in an interaction is a consequence of being part of a speech community. Moreover, group identity is enhanced by this shared communicative behavior through the practice of joking. The offensive use of insults, on the contrary, can lead to the development of oppositional identities. Two people offensively insulting each other each underline negative characteristics of the other, in order to distance each person's own identity from the other oppositional one that deserves to be insulted. For an outsider to the speech community of Italian young adults, these uses and their meanings might not be self-evident. Therefore, this study provides an overview of them.

These findings are a product of ethnography and are the result of samples and data derived from participant observations and interviews. They are constructed to be representative of my participants' points of view, as the theoretical framework, EoC, requires. To enhance the dependability of the study, all the steps taken in the process of this study have been properly documented, including initial notes and coding which are not included extensively in this document. The dependability of a qualitative study is guaranteed when anybody can keep track of the steps followed by 
the ethnographer and thus understand how the study was executed from beginning to end (Shenton 2004).

The study, however, suffered some limitations. The number of participants is relatively small to be able to claim that its results are generalizable. The fact that this study has been conducted in Madrid, Spain, and not in Italy definitely influenced the potential number of interviewees and direct observations and also the fact that the use of insults by Italians in Spain may be different compared to the use of insults by Italians in Italy. However, my background as an Italian and my regular visits to Italy allowed me to confirm that there were no apparent differences in the use of insults by Italians in both countries.

The study cannot be compared to any other study on the same topic, as there are none. However, future research can be conducted on similar or related topics. For instance, it would be interesting to further investigate Giulia's contradicting point of view on gender identities. In addition, a similar study conducted on youth would enrich the literature even more, as it seems like the use of insults is a communicative behavior more typical in this age group. 


\section{Appendix}

Table 1 - Initial and potential themes These themes were identified by "sorting the different codes ... and collating all the relevant coded data extracts within the identified themes" (Braun and Clarke 2006, 89). Some related codes out of the 55 identified are listed as well.

\begin{tabular}{|c|c|c|c|}
\hline \multicolumn{3}{|c|}{$\begin{array}{l}\text { Potential/Initial } \\
\text { Themes }\end{array}$} & \multirow{2}{*}{$\begin{array}{l}\text { Related Codes } \\
\text { In the past; }\end{array}$} \\
\hline 1) & Insults are for & a. & \\
\hline & & b. & Lady; \\
\hline & & c. & Manly. \\
\hline \multirow[t]{4}{*}{ 2) } & Depends on; & a. & Family and School; \\
\hline & & b. & Manual job; \\
\hline & & c. & $\begin{array}{l}\text { Persons (boss, friends, } \\
\text { subordinates, } \\
\text { unknown people, etc.); }\end{array}$ \\
\hline & & d. & Geographical Area. \\
\hline \multirow[t]{2}{*}{ 3) } & Habit / Routine; & a. & Frequent; \\
\hline & & b. & Habit. \\
\hline \multirow[t]{2}{*}{ 4) } & Use; & a. & $\begin{array}{l}\text { Light (nickname, joke, } \\
\text { friendly, etc.); }\end{array}$ \\
\hline & & b. & $\begin{array}{l}\text { Offensive (to offend, } \\
\text { reaction, not } \\
\text { belonging, etc.). }\end{array}$ \\
\hline \multirow[t]{3}{*}{ 5) } & Identity. & a. & Young age; \\
\hline & & b. & Regret; \\
\hline & & c. & Meanings. \\
\hline
\end{tabular}

Table 2 - Final themes and sub-themes identified through the analysis. The sub-themes listed in this table are not the only one identified in the analysis, but they are the most relevant and pivotal in understanding the value of the four main themes.

\begin{tabular}{|c|c|c|}
\hline Final themes & & Sub-themes \\
\hline Depending on Gender & a. & Masculine Identity; \\
\hline \multirow{3}{*}{$\begin{array}{l}\text { Depending on Social } \\
\text { Context }\end{array}$} & a. & Age; \\
\hline & & Education; \\
\hline & & Workplace. \\
\hline $\begin{array}{l}\text { Through the Light Use } \\
\text { of Insults }\end{array}$ & a. & Confidenza. \\
\hline $\begin{array}{l}\text { Through the Offensive } \\
\text { Use of Insults }\end{array}$ & a. & $\begin{array}{l}\text { Oppositional } \\
\text { Identity. }\end{array}$ \\
\hline
\end{tabular}




\section{References}

Alderete, Ethel, Madalena Monteban, Steve Gregorich, Celia P.

Kaplan, Raul Mejia, and Eliseo J. Perez-Stable. 2012. “Smoking and Exposure to Racial Insults among Multiethnic Youth in Jujuy, Argentina." Cancer Causes \& Control 23, (February): 37-44.

Braun, Virginia, and Victoria Clarke. 2006. “Using thematic analysis in psychology." Qualitative Research in Psychology 3(2): 77-101.

Donovan-Kicken, Erin, Andrew C. Tollison, and Elizabeth S. Goins. 2011. "A Grounded Theory of Control over Communication Among Individuals with Cancer." Journal of Applied Communication Research 39(3): 310-330.

Emerson, Robert M., Rachel I. Fretz, and Linda L. Shaw. 2011. "Pursuing Members' Meanings." In Writing Ethnographic Fieldnotes (2nd ed.), 129-169. Chicago: University of Chicago Press.

Flynn, Charles P. 1976. "Sexuality and Insult Behavior." The Journal of Sex Research 12(1): 1-13.

Hymes, Dell. 1972. "Models of the interaction of language and social life." In Directions in Sociolinguistics: The Ethnography of Communication, edited by John J. Gumperz and Dell Hymes, 3571. New York: Holt, Rinehart and Winston.

Hymes, Dell. 1974. "Ways of speaking." In Explorations in the Ethnography of Speaking, edited by Richard Bauman and Joel Sherzer, 433-451. Cambridge: Cambridge University Press.

Kegl, Rosemary. 1994. "'The Adoption of Abominable Terms': The Insults That Shape Windsor's Middle Class." ELH61(2): 253-278.

Kiesling, Scott F. 2001. '"Now I Gotta Watch What I Say': Shifting Constructions of Masculinity in Discourse." Journal of Linguistic Anthropology 11(2): 250-273.

Littlejohn, Stephen W., Karen A. Foss, and John G. Oetzel. 2016. Theories of Human Communication. Waveland Press.

Murray, Stephen O. 1979. "The Art of Gay Insulting." Anthropological Linguistics 21(5): 211-223.

Philipsen, Gerry. 1992. Speaking Culturally: Explorations in Social Communication. Albany: State University of New York Press. 
Philipsen, Gerry, and Lisa M. Coutu. 2005. "The Ethnography of Speaking." In The Handbook of Language and Social Interaction, edited by Kristine L. Fitch and Robert E. Sanders, 355-379. Mahwah, NJ: Lawrence Erlbaum Associates.

Samarin, William. 1969. "The Art of Gbeya Insults." International Journal of American Linguistics 35, no. 4: 323-329.

Shenton, Andrew K. 2004. "Strategies for ensuring trustworthiness in qualitative research projects." Education for Information 22(2): 63 -75 .

Tartamella, Vito. 2011. “Contare le Parolacce." Accessed May 2, 2018. https://www.parolacce.org/2011/12/18/contare-le-parolacce/

Tartamella, Vito. 2016. "Parolacce." Self-published eBook. https:// www.parolacce.org/2016/08/10/parolacce-formato-ebook/

Tartamella, Vito. 2016. "Parolacce: le Più Amate dagli Italiani." Accessed May 2, 2018.

Tracy, Sarah J. 2013. Qualitative Research Methods: Collecting Evidence, Crafting Analysis, Communicating Impact. Hoboken, NJ: Wiley-Blackwell.

Wilkins, Richard J. 2009. “The Asiasta Puhuminen event." In Speech Culture in Finland, edited by Richard Wilkins and Pekka Isotalus, 63-84. New York: University Press of America.

Witteborn, Saskia, and Qian Huang. 2017. “Diaosi [underdog talk] as a way of relating in contemporary China." In The Handbook of Communication in Cross-Cultural Perspective, edited by Donal Carbaugh, 142-152. New York: Routledge. 